\title{
How do we measure success and at the same time meet patient expectations?
}

\author{
Klaus Bak ${ }^{1}$ Albert Lin ${ }^{2}$
}

Received: 7 August 2017 / Accepted: 21 August 2017 / Published online: 13 September 2017

(c) European Society of Sports Traumatology, Knee Surgery, Arthroscopy (ESSKA) 2017

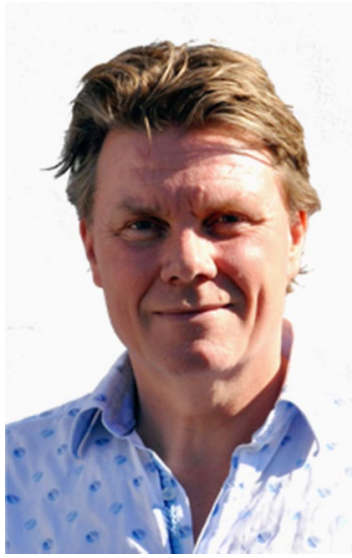

Klaus Bak

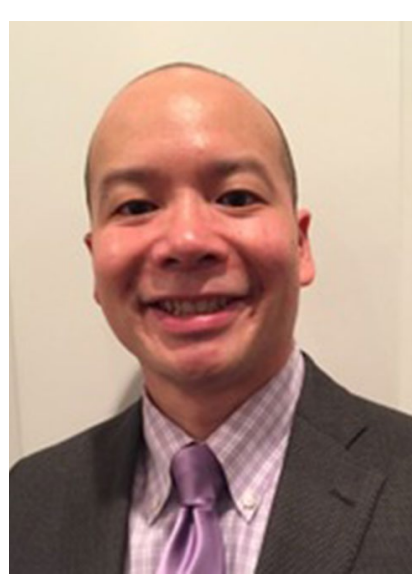

Albert Lin
In the current issue of our journal, Plath et al. [3] present a paper on the expectations of patients undergoing surgery for glenohumeral instability. They raise the question that despite a successful operation from the surgeon's point of view, unrealistic patient expectations may lead to dissatisfaction. In the end, this disconnect makes no one happy.

The first and most widely used score for shoulder instability was the Rowe score which was solely based on objective measures. As with other outcome measures from the 1980's, the score was not validated but rapidly used worldwide and generally accepted as the best shoulder instability outcome

Klaus Bak

skulderbak@gmail.com

Kysthospitalet, Skodsborg, Denmark

2 Division of Shoulder Surgery and Sports Medicine, University of Pittsburgh, UPMC Center for Sports Medicine, Pittsburgh, USA score. Despite its lack of scientific validation, the Rowe score gained global popularity due to the possibility of comparing outcome results of shoulder instability surgery. About 20 years ago, the new consensus was that the best outcome measurement tools should be a combination of the patient's subjective experience and the investigators objective data, such as the Constant-Murley score which consists of $65 \%$ objective data and $35 \%$ subjective assessment. During the last 10-20 years, there has been an increasing understanding that even with complete correction of pathology from an objective radiographic and physical exam standpoint, patient satisfaction may not correlate with these measures of "success" following surgical stabilization. In the search for a $100 \%$ patient-related outcome measure, Kirkley et al. [1] from Western Ontario in Canada developed the Western Ontario Shoulder Instability Index which is now one of most used and best validated shoulder instability outcome scores. Plancher and Lipnick [2] evaluated 10 different scoring systems and found that the Melbourne Instability Shoulder Score (MISS) and Western Ontario Shoulder Instability Index (WOSI) had the highest response rate and inter-rater reliability. Today, patient-reported outcome measures (PROMs) are the gold standard, but despite scientific validation these scores rarely evaluate or incorporate patient expectations. Plath et al. [3], in the current issue, follow a cohort of patients undergoing arthroscopic Bankart with emphasis on the patients' expectations through a novel, but non-validated outcome measure, and found that the patients' expectations for both primary and revision shoulder instability repair were inflated, highlighting that there may still be a significant discordance between what surgeons and patients consider to be a successful outcome.

The twenty-first century in healthcare has ushered an era of emphasis on value driven care and quality metrics as benchmarks of success and reimbursement. As such, the 
definition of what constitutes quality is increasingly trending towards a more holistic approach of treatment that includes patient perception and expectations as major components of a successful outcome. More steps can always be taken towards the ideal treatment. The correct diagnosis, anatomic correction of the pathology, the correct rehabilitation, and the correct timing of return to sports, especially for athletes remain, of course, important considerations. Yet, even when all of these parameters are met, we must remain astutely aware of the patient's expectations and whether postoperative results match the preoperative perceptions. An appropriate understanding of expectations can only be adequately achieved before treatment is initiated, when there is an opportunity to counsel the patient and participate in shared decision-making. To mitigate surgeon and patient discrepancy, emphasis may be shifted towards a more thorough preoperative discussion that includes the consequences of the treatment and the best evidence-based recommendations regarding course, prognosis, benefits and drawbacks. Lastly, scientific, well-validated PROMs emphasizing patient expectations should also be developed and are necessary to accurately measure preoperative expectation.

\section{References}

1. Kirkley A, Griffin S, McLintock H, Ng L (1998) The development and evaluation of a disease-specific quality of life measurement tool for shoulder instability. The Western Ontario Shoulder Instability Index (WOSI). Am J Sports Med 26(6):764-772

2. Plancher KD, Lipnick SL (2009) Analysis of evidence-based medicine for shoulder instability. Arthroscopy 25(8):897-908

3. Plath JE, Saier T, Feucht MJ, Minzlaff P, Seppel G, Braun S, Hatch MD, Imhoff AB (2017) Patients' expectations of shoulder instability repair. Knee Surg Sports Traumatol Arthrosc. doi:10.1007/s00167-017-4489-7 